\title{
The Convergence of Spline Collocation for Strongly Elliptic Equations on Curves *
}

\author{
Dedicated to Prof. Dr. Dr. h.c. mult. Lothar Collatz \\ on the occasion of his 75 th birthday \\ Douglas N. Arnold ${ }^{1}$ and Wolfgang L. Wendland ${ }^{2}$ \\ 1 Department of Mathematics, University of Maryland, College Park, MD 20742, USA \\ ${ }^{2}$ Fachbereich Mathematik, Technische Hochschule, D-6100 Darmstadt, Federal Republic of Ger- \\ many
}

\begin{abstract}
Summary. Most boundary element methods for two-dimensional boundary value problems are based on point collocation on the boundary and the use of splines as trial functions. Here we present a unified asymptotic error analysis for even as well as for odd degree splines subordinate to uniform or smoothly graded meshes and prove asymptotic convergence of optimal order. The equations are collocated at the breakpoints for odd degree and the internodal midpoints for even degree splines. The crucial assumption for the generalized boundary integral and integro-differential operators is strong ellipticity. Our analysis is based on simple Fourier expansions. In particular, we extend results by J.Saranen and W.L. Wendland from constant to variable coefficient equations. Our results include the first convergence proof of midpoint collocation with piecewise constant functions, i.e., the panel method for solving systems of Cauchy singular integral equations.
\end{abstract}

Subject Classifications: AMS(MOS): 65R20, 65N99, 65N30, 65E05, 30C30, 73K30, 65N35; CR: G.1.9.

\section{Introduction}

Spline collocation methods are extensively employed for the numerical solution of a variety of integral, differential, and integro-differential equations (or, more generally, pseudodifferential equations $[47,48]$ ) posed on plane curves. In fact, collocation is the most widely used numerical technique for solving the bound-

* This work was begun at the Technische Hochschule Darmstadt where Professor Arnold was supported by a North Atlantic Treaty Organization Postdoctoral Fellowship. The work of Professor Arnold is supported by NSF grant BMS-8313247. The work of Professor Wendland was supported by the "Stiftung Volkswagenwerk" 
ary integral equations arising from exterior or interior boundary value problems of elasticity, fluid dynamics, electromagnetics, acoustics, and other engineering applications with the boundary element technique. See, for example, the books and conference proceedings on the boundary element method $[6,7,9-13,15,19,27]$, and the discussion of the boundary integral equations arising in applications in [3]. Despite their prevalance, however, no general approach to the error analysis of spline collocation methods was known until recently. Convergence had been shown only in special cases - the most important being the case of Fredholm integral equations of the second kind (see, e.g. $[4,5,14,20,31,32]$ - and the methods of analysis generally depended quite strongly on the particular form of the equation considered.

Quite recently two more general techniques of analysis have been introduced, the first in the case of collocation by odd degree splines at the nodal points, the second in the case of collocation by even degree splines at the internodal midpoints. The first method is due to Arnold and Wendland [3]. It is based on equivalence of the collocation method with a mesh dependent Galerkin-Bubnov method ${ }^{1}$ and is quite general, yielding optimal asymptotic rates of convergence in the whole range of Sobolev spaces $H^{s}$ for which they hold. This method applies as long as the equation is strongly elliptic, a condition which is also known to be necessary for convergence in most cases $[36,37,43]$. The combination of the equivalence mentioned above and strong ellipticity permits an error analysis analogous to that for a standard Galerkin method. Unfortunately, it does not appear possible to extend this method of analysis to the even degree case. Therefore Saranen and Wendland [39] use another approach to obtain results similar to those of [3]. Although they show the equivalence of the collocation equations with certain Galerkin-Petrov equations, the heart of their analysis is not this equivalence but rather simple Fourier analysis techniques. Consequently, this second method entails two significant restrictions. First, the operator, in addition to being strongly elliptic, must have a principal part with constant coefficients. Second, the spacing of the knots of the splines is required to be uniform, in contrast to the analysis of [3] in the odd degree case, for which no restriction on mesh spacing was needed.

For the special case of piecewise linear spline collocation on Cauchy singular integral equations with smooth coefficients, a different method has been developed by Prössdorf and Schmidt in [36, 37] which has been extended to piecewise continuous coefficients by Prössdorf and Rathsfeld in [34, 35].

Since the appearance of [3] and [39], G. Schmidt has extended these analyses in various ways. In [41] he analyzes nodal collocation by even order splines for a different sort of (non strongly elliptic) singular integral operators and for the corresponding pseudodifferential operators in [43]. In a recent paper [42] he considers spline collocation on a uniform mesh for operators with constant coefficients using collocation points which are displaced from the nodes or internodal midpoints.

In this paper we present an analysis which treats the odd and even degree cases together by exploiting the Fourier series expansions of the splines and

1 By a Galerkin-Bubnov method we mean a Galerkin method with equal test and trial spaces, while a Galerkin-Petrov method permits distinct spaces 
the equations (an approach which for the identity operator goes back to Quade and Collatz [51]). For odd degree splines the results improve only slightly on those of [3] (e.g., estimates are given for a less regular solution than permitted in [3]). Moreover the knot spacing permitted is restricted, so in this respect the results are inferior to those of [3]. In the even order case, however, we remove the requirement from [39] that the principal part has constant coefficients, allowing instead any sufficiently smooth coefficients. This is our principal new result. It shows that collocation by even degree splines at the midpoints converges with optimal order for the same class of equations as the ordinary spline Galerkin-Bubnov method and odd degree spline collocation (namely, for strongly elliptic equations). We have had less success in removing the restriction of uniform knot spacing. However we at least relax this requirement to allow smoothly graded partitions, i.e., partitions which are mapped onto uniform partitions by a smooth diffeomorphism. Also the present results are more general than heretofore known in one other respect. In both [3] and [39] it is assumed that the degree of the splines exceeds the order of the equation. Since this condition is equivalent to the requirement that the image of every spline under the operator be continuous, and since the collocation method requires that the point values at the collocation points of these images be defined, this appears to be a natural condition. However the discontinuity in the image of a spline can occur only at the knots, and these coincide with the collocation points only in the odd degree case. In the even degree case we show that this condition can be relaxed to allow splines with degree exceeding $\beta-1 / 2$ where $\beta$ is the order of the equation. Consequently the present analysis applies to situations excluded previously. In particular the present work provides the first convergence proof of the panel method, i.e., midpoint collocation with piecewise constant functions, for solving Cauchy singular integral equations. This method is widely used, $[8,9,16,28,38,49]$, as well as collocation with higher degree splines.

In order to state our results more precisely we now introduce some notation. We shall consider the numerical solution of the equation $L u=F$ where $F$ is a given continuous 1-periodic function and $u$ is a 1-periodic function which we seek. (It is only for simplicity of notation that we consider a single equation. As in [3] our results easily extend to systems of equations. Via parametric representations of the curves as in [3] a system of equations on one or more simple closed curves is thus covered.) Each periodic distribution $u$ has a Fourier expansion

$$
u(x)=\sum_{k \in \mathbb{Z}} \hat{u}(k) e^{2 \pi i k x}
$$

where the Fourier coefficients are given by the formula

$$
\hat{u}(k)=\int_{0}^{1} u(x) e^{-2 \pi i k x} d x
$$

in case $u$ is locally integrable. For $s \in \mathbb{R}$ define the inner product

$$
(u, v)_{s}=\hat{u}(0) \bar{v}(0)+\sum_{k \neq 0}|2 \pi k|^{2 s} \hat{u}(k) \bar{v}(k) .
$$


The Sobolev space $H^{s}$ (all function spaces are supposed, without special notation, to be complex valued and periodic) consists of all periodic distributions $u$ for which the norm $\|u\|_{s}:=\sqrt{(u, u)_{s}}$ is finite. For $\beta \in \mathbb{R}$ we define operators $Q_{+}^{\beta}$ and $Q_{-}^{\beta}$ by

$$
\begin{aligned}
& Q_{+}^{\beta} u(x)=\sum_{k \neq 0} \mid k^{\beta} \hat{u}(k) e^{2 \pi i k x}, \\
& Q_{-}^{\beta} u(x)=\sum_{k \neq 0} \operatorname{sign}(k)|k|^{\beta} \hat{u}(k) e^{2 \pi i k x} .
\end{aligned}
$$

For all $s \in \mathbb{R}, Q_{ \pm}^{\beta}$ maps $H^{s}$ boundedly into $H^{s-\beta}$. For $\beta=0, Q_{+}^{0} u+\hat{u}(0)=u$ and $Q_{-}^{0} u+\hat{u}(0)$ is the Hilbert transform of $u$.

The operators considered here are of the form

$$
L u(x)=b_{+}(x) Q_{+}^{\beta} u(x)+b_{-}(x) Q_{-}^{\beta} u(x)+K u(x)
$$

where $\beta \in \mathbb{R}$ is the order of the operator, $b_{+}$and $b_{-}$are functions in $C^{\infty}(\mathbb{R})$, and for all $r \in \mathbb{R}, K$ maps $H^{r}$ boundedly into $H^{r-\beta+\delta}$ for some $\delta>0$. (We actually require this property of $K$ only for a limited range of $r$, and require only finitely many derivatives of $b_{ \pm}$. The exact requirements can be ascertained by a close examination of the arguments in Sects. 3-5.) We say that $L$ is strongly elliptic if there exists a smooth periodic function $\theta$ such that

$$
\gamma:=\inf _{x \in \mathbb{R}} \min \left\{\operatorname{Re} \theta(x)\left[b_{+}(x)+b_{-}(x)\right], \operatorname{Re} \theta(x)\left[b_{+}(x)-b_{-}(x)\right]\right\}>0 .
$$

In this case $L: H^{s} \rightarrow H^{s-\beta}$ is a Fredholm operator of index zero for all $s \in \mathbb{R}$, and if $L$ is injective for some $s$ it is in fact bijective for all $s$. We shall assume that $L$ is injective. (In the case of a system of Eqs. (1.1) and (1.2) must be modified as follows. In (1.1) $u$ represents a vector of functions, i.e., an element of the Cartesian product $\left(H^{s}\right)^{p}$ with $p>1, Q_{ \pm}^{\beta}$ is understood to act on each component, and $b_{+}$and $b_{-}$are $p \times p$ matrices of functions. The strong ellipticity condition is then

$$
\gamma:=\inf \min \left\{\operatorname{Re}\left[\zeta^{T} \theta(x)\left(b_{+}(x)+b_{-}(x)\right) \bar{\zeta}\right], \operatorname{Re}\left[\zeta^{\top} \theta(x)\left(b_{+}(x)-b_{-}(x)\right) \bar{\zeta}\right]\right\}>0,
$$

where now $\theta$ is matrix valued and the infimum is over $x \in \mathbb{R}$ and unit vectors $\zeta \in \mathbb{C}^{p}$.)

Let $d$ be a nonnegative integer, $n$ a natural number, $h=1 / n$. Set $\Delta$ $=\{j h \mid j \in \mathbb{Z}\}$,

$$
\Xi= \begin{cases}\Delta, & d \text { odd } \\ \{(j+1 / 2) h \mid j \in \mathbb{Z}\}, & d \text { even. }\end{cases}
$$

Let $\mathscr{S}_{h}^{d}$ denote the space of smooth splines of degree $d$ on the uniform mesh $\Xi$. Thus $\mathscr{S}_{h}^{d}$ consists of periodic $C^{d-1}$ piecewise polynomials of degree $d$ and has dimension $n$. The collocation method determines an approximate solution $u_{h} \in \mathscr{S}_{h}^{d}$ to the operator equation

by the equations

$$
L u=F \quad \text { on } \mathbb{R}
$$

$$
L u_{h}=F \quad \text { on } \Delta
$$


Once a basis for $\mathscr{S}_{h}^{d}$ is chosen, (1.4) is easily reduced to an $n \times n$ linear system for the unknown coefficients of $u_{h}$.

We can now state our main result in the case of uniform knot spacing.

Theorem 1.1. Let $\beta$ be a real number and $L$ a strongly elliptic, injective operator of order $\beta$ having the form (1.1). Let $d$ be either a positive odd integer exceeding $\beta$ or a nonnegative even integer exceeding $\beta-1 / 2$. Then there exists $h_{0}>0$ such that for $0<h \leqq h_{0}$ and any continuous function $F$ the collocation equations (1.4) are uniquely solvable for $u_{h} \in \mathscr{S}_{h}^{d}$. Moreover, if $s, t \in \mathbb{R}$ satisfy

$$
\beta \leqq s \leqq t \leqq d+1, \quad s<d+1 / 2, \quad \beta+1 / 2<t,
$$

and the solution $u$ to (1.3) is in $H^{t}$, then there holds the optimal error estimate

$$
\left\|u-u_{h}\right\|_{s} \leqq C h^{t-s}\|u\|_{t} .
$$

Here and in the following $C$ denotes a generic constant independent of $h$ and $u$. In (1.6) $C$ may depend on an upper bound for the magnitudes of the coefficients and their derivatives, a lower bound for the strong ellipticity constant $\gamma$, and on $\beta, d, s$, and $t$.

Let us comment on the hypotheses of the theorem. The strong ellipticity condition (1.2) is essential, as remarked above. In [26, p. 205] an example is given of an elliptic, but not strongly elliptic, singular integral equation for which nodal collocation with piecewise linear splines diverges.

The hypotheses (1.5) on $s$ and $t$ are essentially as weak as possible. The condition $s \leqq t \leqq d+1$ is clearly required from approximation theory and the condition $s<d+1 / 2$ is required so that the left hand side of (1.6) makes sense. The collocation does not converge with optimal order in $H^{s}$ for $s<\beta$, as it can be shown that the error is no smaller than $O\left(h^{d+1-\beta}\right)$ in any Sobolev space, cf. [3]. Finally we cannot allow $t \leqq \beta+1 / 2$ since then for a general $u \in H^{t}, F=L u$ will be discontinuous and so we cannot sensibly collocate.

Operators of the form (1.1), although they may appear to be rather special, form a rather general class, including all pseudo-differential operators on the circle [1] (and so, via parametrization, on closed curves). Many important examples of strongly elliptic operators of this form arise from boundary integral methods, e.g., singular integral equations and hypersingular equations arising from acoustics, fluid dynamics, elasticity, and quantum field theory. (For further details and numerous other applications see $[3,25,50]$.) Another example is Symm's integral equation of conformal mapping. Our Analysis provides error estimates for the numerical methods for this equation presented in [21] and [46]. Recent numerical experiments by Hoidn [24] show excellent agreement with the theoretical convergence rates for this equation.

The remainder of the paper proceeds as follows. In the next section we prove Theorem 1.1 under the additional assumption that $L$ has constant coefficients, $K$ has a special form, and the meshes are uniform. We use an explicit Fourier analysis as in [39] but our proof is more direct and elementary, gives somewhat sharper results, and enables us to consider odd and even degree splines at the same time. In Sect. 3 we remove the assumption of constant coefficients for $L$ by locally freezing coefficients and using perturbation tech- 
niques familiar from the existence theory for partial differential equations (Korn's trick). (Independently Prössdorf [33] recently applied a localization principle for spline approximations of pseudodifferential equations which is closely related to our technique.) All necessary analysis is presented by using only Fourier expansions.

In Sect. 4 we extend our analysis to smoothly graded partitions. Here, however, we need one special property of pseudo-differential operators, Theorem 4.2 , for which we have not been able to find an elementary proof. Finally Sect. 5 collects proofs and references for various elementary lemmas.

\section{Convergence of the Collocation Method for Operators with Constant Coefficients}

We now prove Theorem 1.1 under the additional restriction that $L$ has the form

$$
L_{0}=b_{+} Q_{+}^{\beta}+b_{-} Q_{-}^{\beta}+J,
$$

where $J u:=\int_{0}^{1} u d x$ and $b_{+}, b_{-}$are complex constants satisfying the strong ellipticity condition

$$
y:=\min \left\{\operatorname{Re}\left(b_{+}+b_{-}\right), \operatorname{Re}\left(b_{+}-b_{-}\right)\right\}>0 .
$$

Note that

$$
\widehat{L_{0} u}(m)= \begin{cases}\left(b_{+}+\operatorname{sign}(m) b_{-}\right)|m|^{\beta} \hat{u}(m), & m \neq 0, \\ \hat{u}(0), & m=0 .\end{cases}
$$

It follows easily from (2.2), Parseval's identity, the definition of the norm in $H^{s+\beta}$, and (2.1) that

$$
\min \left(1, \gamma /(2 \pi)^{\beta}\right)\|v\|_{s+\beta} \leqq\left\|L_{0} v\right\|_{s} \leqq \max \left(1,\left(\left|b_{+}\right|+\left|b_{-}\right|\right) /(2 \pi)^{\beta}\right)\|v\|_{s+\beta} .
$$

We shall analyze the collocation method using Fourier series. To this end we first reformulate the collocation equations in terms of the Fourier coefficients. The key result is given in the following lemma. We use the notation $\equiv$ to indicate congruence modulo $n$, and set $A_{n}=\left\{p \in \mathbb{Z} \mid-\frac{n}{2}<p \leqq \frac{n}{2}\right\}$, a set of coset representatives modulo $n$. The notation $\sum_{m}$ denotes $\lim _{i \rightarrow \infty} \sum_{m=-l}^{l}$ and $\sum_{m \equiv g}$ denotes $\lim _{l \rightarrow \infty} \sum_{\substack{m=-l \\ m \equiv p}}^{l}$

Lemma 2.1. Let $\phi \in L^{1}$ and suppose that $\phi$ is Hölder continuous in some neighborhood of 4 . Then $\sum_{m \equiv p} \hat{\phi}(m)<\infty$ for all $p$. Moreover

$$
\sum_{m \equiv p} \hat{\phi}(m)=0 \quad \text { for all } p \in \Lambda_{n}
$$


if and only if

$$
\phi=0 \quad \text { on } \Delta .
$$

Proof. The hypotheses imply that the Fourier series for $\phi$ converges to $\phi(q / n)$ at $x=q / n$ :

$$
\phi(q / n)=\sum_{m} \hat{\phi}(m) e^{2 \pi i m q / n} .
$$

Hence

$$
\begin{aligned}
\frac{1}{n} \sum_{q \in \Lambda_{n}} \phi(q / n) e^{-2 \pi i p q / n} & =\frac{1}{n} \sum_{q \in \Lambda_{n}} \sum_{m} \hat{\phi}(m) e^{2 \pi i(m-p) q / n} \\
& =\sum_{m} \hat{\phi}(m) \frac{1}{n} \sum_{q \in \Lambda_{n}} e^{2 \pi i(m-p) q / n}=\sum_{m \equiv p} \hat{\phi}(m) .
\end{aligned}
$$

Since the Vandermonde matrix $\left(e^{-2 \pi i p q / n}\right)_{p, q \in A_{n}}$ is nonsingular, the lemma follows.

As a corollary we get the following proposition.

Proposition 2.2. Let $u \in H^{t}$ for some $t>\beta+1 / 2$ and let $u_{h} \in \mathscr{S}_{h}^{d}$ where $d$ is either a positive odd integer exceeding $\beta$ or a nonnegative even integer exceeding $\beta-1 / 2$. Then the collocation equations

$$
L_{0} u_{h}(x)=L_{0} u(x), \quad x \in \Delta,
$$

are satisfied if and only if

$$
\sum_{m \equiv p} \widehat{L_{0} u}(m)=\sum_{m \equiv p} \widehat{L_{0} u_{h}}(m), \quad p \in \Lambda_{n} .
$$

Since $L_{0} u \in H^{t-\beta}$ and $t-\beta>1 / 2$, the Sobolev embedding theorem implies that $L_{0} u$ is Hölder continuous. Moreover $\mathscr{S}_{h}^{d} \subset H^{s}$ for all $s<d+1 / 2$ so the hypotheses imply that $L_{0} u_{h}$ is Hölder continuous if $d$ is odd and in any case $L_{0} u_{h} \subset L^{2}$. When $d$ is even $u_{h}$ is smooth in a neighborhood of $\Delta$, whence $L_{0} u_{h}$ is Hölder continuous in a neighborhood of $\Delta$ (see Lemma 3.2c). Hence the sums appearing in (2.5) converge and the result follows by applying the lemma with $\phi=L_{0} u_{h}-L_{0} u$.

In order to apply Proposition 2.2 we employ (2.2) to observe that for any $u$ with $L_{0} u$ integrable and Hölder continuous in a neighborhood of $\Delta$,

$$
\sum_{m \equiv p} \widehat{L_{0} u}(m)=b_{0}(p) \hat{u}(p)+\sum_{k \in \mathbb{Z}^{*}}\left[b_{+}+\operatorname{sign}(k) b_{-}\right]|p+k n|^{\beta} \hat{u}(p+k n), \quad p \in \Lambda_{n},
$$

where

$$
b_{0}(p):= \begin{cases}{\left[b_{+}+\operatorname{sign}(p) b_{-}\right]|p|^{\beta},} & p \neq 0, \\ 1, & p=0 .\end{cases}
$$

(The asterisk appended to a set of integers denotes the complement of zero in the set. Thus $\mathbb{Z}^{*}=\mathbb{Z} \backslash\{0\}$.)

For the right hand side of (2.5) we combine (2.6) with the recursion relation for the Fourier coefficients of a spline function:

$$
\hat{\phi}(p+k n)(p+k n)^{d+1}=(-1)^{k(d+1)} \hat{\phi}(p) p^{d+1}, \quad p, k \in \mathbb{Z}, \phi \in \mathscr{S}_{h}^{d} .
$$


(For a proof of the recursion relation see, e.g., (41) in [51] and [2].) Thus, for $u_{h} \in \mathscr{S}_{h}^{d}, p \in \Lambda_{h}$,

$$
\begin{aligned}
\sum_{m \equiv p} \widehat{L_{0} u_{h}}(m)= & b_{0}(p) \hat{u}_{h}(p)+p^{d+1} \sum_{k \in \mathbb{Z}^{*}}\left(b_{+}+\operatorname{sign}(k) b_{-}\right) \\
& \cdot(-1)^{k(d+1)} \operatorname{sign}(k)^{d+1}|p+k n|^{\beta-d-1} \hat{u}_{h}(p) \\
= & \left\{b_{0}(p)+\operatorname{sign}(p)^{d+1}|p|^{\beta}|2 p / n|^{d+1-\beta} \sum_{k \in \mathbb{Z}^{*}}\left(b_{+}+\operatorname{sign}(k) b_{-}\right)\right. \\
& \left.\cdot(-1)^{k(d+1)} \operatorname{sign}(k)^{d+1}|(2 p / n)+2 k|^{\beta-d-1}\right\} \hat{u}_{h}(p) .
\end{aligned}
$$

Defining for $y \in[-1,1]$

$$
\begin{aligned}
& f(y)=\operatorname{sign}(y)^{d+1}|y|^{d+1-\beta} \sum_{k \in \mathbb{Z}^{*}}(-1)^{k(d+1)} \operatorname{sign}(k)^{d+1}|y+2 k|^{\beta-d-1}, \\
& g(y)=-\operatorname{sign}(y)^{d}|y|^{d+1-\beta} \sum_{k \in \mathbb{Z}^{*}}(-1)^{k(d+1)} \operatorname{sign}(k)^{d}|y+2 k|^{\beta-d-1},
\end{aligned}
$$

we may write (2.8) more compactly as

$$
\sum_{m \equiv p}{\widehat{L_{0}}}_{u_{h}}(m)= \begin{cases}|p|^{\beta}\left\{b_{+}[1+f(2 p / n)]+\operatorname{sign}(p) b_{-}[1-g(2 p / n)]\right\} \hat{u}_{h}(p), & p \in \Lambda_{n}^{*}, \\ \hat{u}_{h}(0), & p=0 .\end{cases}
$$

In the following lemma we summarize the elementary properties of $f$ and $g$ which we shall require. They are verified in Sect. 5.

Lemma 2.3. Suppose that $\beta<d$ for $d$ odd and $\beta<d+1$ for $d$ even. Then $f$ and $g$ defined by (2.9) are continuous, even, nonnegative functions on $[-1,1]$ and are strictly increasing on $[0,1]$. Moreover $g(1)=1$ and there exists constant $C$ depending only on $\beta$ and $d$ so that

$$
|f(y)|+|g(y)| \leqq C|y|^{d+1-\beta}, \quad y \in[-1,1] .
$$

We now proceed with the proof of Theorem 1.1 for the operator $L_{0}$. Set

$$
D(y)=[1+f(y)] b_{+}+\operatorname{sign}(y)[1-g(y)] b_{-}, \quad y \in[-1,1] .
$$

Let $s, t$ be as in Theorem 1.1 and $u \in H^{t}$. In light of Proposition 2.2 and (2.10), $L_{0} u_{h}$ collocates $L_{0} u$ (i.e., (2.4) holds) if and only if

$$
\begin{aligned}
|p|^{\beta} D(2 p / n) \hat{u}_{h}(p) & =\sum_{m \equiv p} \widehat{L_{0} u}(m), \quad p \in A_{n}^{*}, \\
\hat{u}_{h}(0) & =\sum_{m \equiv 0} \widehat{L_{0} u}(m) .
\end{aligned}
$$

Note that since

$$
0 \leqq 1-g(y) \leqq 1 \leqq 1+f(y)<\infty, \quad y \in[-1,1],
$$

by Lemma 2.3, it follows from the strong ellipticity condition (2.1) that

$$
\operatorname{Re} D(y) \geqq \gamma>0, \quad y \in[-1,1] \text {. }
$$


In particular the coefficient of $\hat{u}_{h}(p)$ in $(2.11 \mathrm{a})$ does not vanish. Hence the Fourier coefficients $\hat{u}_{h}(p), p \in \Lambda_{h}$, are uniquely determined by (2.11), namely

$$
\begin{aligned}
\hat{u}_{h}(p)= & D(2 p / n)^{-1}|p|^{-\beta} \\
& \cdot\left\{b_{0}(p) \hat{u}(p)+\sum_{k \in \mathbb{Z}^{*}}\left[b_{+}+\operatorname{sign}(k) b_{-}\right]|p+k n|^{\beta} \hat{u}(p+k n)\right\}, \quad p \in \Lambda_{n}^{*}, \\
\hat{u}_{h}(0)= & \hat{u}(0)+\sum_{k \in \mathbb{Z}^{*}}\left[b_{+}+\operatorname{sign}(k) b_{-}\right]|k n|^{\beta} \hat{u}(k n) .
\end{aligned}
$$

Since by (2.7) these coefficients determine $u_{h}$ uniquely, we have proven that there exists a unique solution to the collocation equations (2.4).

It remains to prove the error estimate

$$
\left\|u-u_{h}\right\|_{s} \leqq C n^{s-t}\|u\|_{t} .
$$

Clearly it suffices to bound each of the four following terms by $C n^{2 s-2 t}\|u\|_{t}^{2}$ :

$$
\begin{aligned}
& T_{1}=\left|\hat{u}(0)-\hat{u}_{h}(0)\right|^{2}, \\
& T_{2}=\sum_{m \in \mathbb{Z} \backslash A_{n}}|\hat{u}(m)|^{2}|m|^{2 s}, \\
& T_{3}=\sum_{m \in \mathcal{Z} \backslash A_{n}}\left|\hat{u}_{h}(m)\right|^{2}|m|^{2 s}, \\
& T_{4}=\sum_{p \in A_{n}^{*}}\left|\hat{u}(p)-\hat{u}_{h}(p)\right|^{2}|p|^{2 s} .
\end{aligned}
$$

We remark that the generic constant $C$ in this section depends only on $\beta, d, s, t$, an upper bound for $\left|b_{+}\right|+\left|b_{-}\right|$, and a positive lower bound for $\gamma$.

Recall the hypotheses on $s$ and $t$, namely

$$
\begin{aligned}
& s \geqq \beta, \\
& s<d+1 / 2, \\
& s \leqq t, \\
& t>\beta+1 / 2, \\
& t \leqq d+1 .
\end{aligned}
$$

We shall frequently use the fact that

$$
\sum_{k \in \mathbb{Z}^{*}}|\theta+2 k|^{r}<C(r), \quad r<-1, \theta \in[-1,1] .
$$

To bound $T_{1}$ we use (2.13b), the Schwarz inequality, (2.16) with $r=2(\beta-t)$ (so $r<-1$ by $(2.15 \mathrm{~d})$ ), and (2.15a):

$$
\begin{aligned}
T_{1} & \leqq C\left[\sum_{k \in \mathbb{Z}^{*}}|k n|^{\beta}|\hat{u}(k n)|\right]^{2} \\
& \leqq C n^{2(\beta-t)} \sum_{j \in \mathbf{Z}^{*}}|j|^{2(\beta-t)} \sum_{k \in \mathbf{Z}^{*}}|\hat{u}(k n)|^{2}|k n|^{2 t} \\
& \leqq C n^{2 s-2 t}\|u\|_{t}^{2} .
\end{aligned}
$$


Next since $m \geqq n / 2$ for $m \in \mathbb{Z} \backslash \Lambda_{n}$,

$$
T_{2}=\sum_{m \in \mathbf{Z} \backslash A_{n}}|\hat{u}(m)|^{2}|m|^{2 t}|m|^{2 s-2 t} \leqq C n^{2 s-2 t}\|u\|_{t}^{2} .
$$

To bound $T_{3}$ first apply (2.7) and (2.13a) to get

$$
\begin{aligned}
\left|\hat{u}_{h}(p+\ln )\right|= & \left|\frac{2 p}{n}\right|^{d+1}\left|\frac{2 p}{n}+2 l\right|^{-d-1} \mid D(2 p / n)^{-1}\left\{\left[b_{+}+\operatorname{sign}(p) b_{-}\right] \hat{u}(p)\right. \\
& \left.+|p|^{-\beta} \sum_{k \in \mathbb{Z}^{*}}\left[b_{+}+\operatorname{sign}(k) b_{-}\right]|p+k n|^{\beta} \hat{u}(p+k n)\right\} \mid, \quad p \in \Lambda_{n}^{*}, l \in \mathbb{Z}^{*} .
\end{aligned}
$$

Now, by (2.16) with $r=2 s-2 d-2$, which is less than -1 by $(2.15 b)$ and (2.15e),

$$
\begin{aligned}
\sum_{p \in A_{n}^{*}} \sum_{l \in \mathbb{Z}^{*}}|\hat{u}(p)|^{2}\left|\frac{2 p}{n}\right|^{2 d+2}\left|\frac{2 p}{n}+2 l\right|^{-2 d-2}|p+\ln |^{2 s} \\
\quad=\left|\frac{n}{2}\right|^{2 s-2 t} \sum_{p \in A_{n}^{*}}\left|\frac{2 p}{n}\right|^{2 d+2-2 t}|\hat{u}(p)|^{2}|p|^{2 t} \sum_{l \in \mathbb{Z}^{*}}\left|\frac{2 p}{n}+2 l\right|^{2 s-2 d-2} \\
\leqq C n^{2 s-2 t}\|u\|_{t}^{2} .
\end{aligned}
$$

Also, by the Schwarz inequality, (2.16) with $r=2 s-2 d-2<-1$, (2.16) with $r$ $=2(\beta-t)<-1$, and $(2.15 \mathrm{~d}, \mathrm{e})$, we find

$$
\begin{aligned}
& \left.\sum_{p \in \Lambda_{n}^{*}} \sum_{l \in \mathbf{Z}^{*}}\left|\frac{2 p}{n}\right|^{2 d+2}\left|\frac{2 p}{n}+2 l\right|^{-2 d-2}|p|^{-2 \beta}\left\{\sum_{k \in \mathbb{Z}^{*}}|p+k n|^{\beta}|\hat{u}(p+k n)|\right\}\right|^{2}|p+l n|^{2 s} \\
& \leqq C n^{2 s-2 t} \sum_{p \in \Lambda_{n}^{*}}\left|\frac{2 p}{n}\right|^{-2 \beta+2 d+2}\left(\sum_{l \in \mathbf{Z}^{*}}\left|\frac{2 p}{n}+2 l\right|^{2 s-2 d-2}\right)\left(\sum_{j \in \mathbb{Z}^{*}}\left|\frac{2 p}{n}+2 j\right|^{2(\beta-t)}\right) \\
& \quad \cdot \sum_{k \in \mathbf{Z}^{*}}|\hat{u}(p+k n)|^{2}|p+k n|^{2 t} \\
& \leqq C n^{2 s-2 t} \sum_{p \in \Lambda_{n}^{*}} \sum_{k \in \mathbf{Z}^{*}}|\hat{u}(p+k n)|^{2}|p+k n|^{2 t} \leqq C n^{2 s-2 t}\|u\|_{t}^{2} .
\end{aligned}
$$

Since $\hat{u}_{h}(l n)=0$ for $l \in \mathbb{Z}^{*}$ by (2.7) we may collect (2.17)-(2.19) and use (2.12) to find that

as desired.

$$
T_{3}=\sum_{p \in \Lambda_{n}^{*}} \sum_{l \in \mathbf{Z}^{*}}\left|\hat{u}_{h}(p+\ln )\right|^{2}|p+\ln |^{2 s} \leqq C n^{2 s-2 t}\|u\|_{t}^{2},
$$

It remains to bound $T_{4}$. From (2.13a), (2.6), and the definitions of $b_{0}$ and $D$ we have for $p \in \Lambda_{n}^{*}$ that

$$
\begin{aligned}
\hat{u}(p)-\hat{u}_{h}(p)= & \hat{u}(p)-D(2 p / n)^{-1}|p|^{-\beta}\left\{b_{0}(p) \hat{u}(p)\right. \\
& \left.+\sum_{k \in \mathbf{Z}^{*}}\left[b_{+}+\operatorname{sign}(k) b_{-}\right]|p+k n|^{\beta} \hat{u}(p+k n)\right\} \\
= & D(2 p / n)^{-1}\left\{\left[f(2 p / n) b_{+}-\operatorname{sign}(p) g(2 p / n) b_{-}\right] \hat{u}(p)\right. \\
& \left.-|p|^{-\beta} \sum_{k \in \mathbf{Z}^{*}}\left[b_{+}+\operatorname{sign}(k) b_{-}\right]|p+k n|^{\beta} \hat{u}(p+k n)\right\} .
\end{aligned}
$$


Now, by (2.12), Lemma 2.3 , and $(2.15 \mathrm{a}, \mathrm{e})$,

$$
\begin{aligned}
& \left|D(2 p / n)^{-1}\left[f(2 p / n) b_{+}-\operatorname{sign}(p) g(2 p / n) b_{-}\right] \hat{u}(p)\right|^{2}|p|^{2 s} \\
& \quad \leqq C n^{2 s-2 t}\left|\frac{2 p}{n}\right|^{2(d+1-\beta+s-t)}|\hat{u}(p)|^{2}|p|^{2 t} \leqq C n^{2 s-2 t}|\hat{u}(p)|^{2}|p|^{2 t}
\end{aligned}
$$

For the remaining terms from (2.20) we have,

$$
\begin{aligned}
& \left\{D(2 p / n)^{-1}|p|^{-\beta} \sum_{k \in \mathbb{Z}^{*}}\left[b_{+}+\operatorname{sign}(k) b_{-}\right]|p+k n|^{\beta}|\hat{u}(p+k n)|\right\}^{2}|p|^{2 s} \\
& \quad \leqq C n^{2 s-2 t}\left|\frac{2 p}{n}\right|^{2(s-\beta)} \sum_{j \in \mathbb{Z}^{*}}\left|\frac{2 p}{n}+2 j\right|^{2(\beta-t)} \times \sum_{k \in \mathbb{Z}^{*}}|\hat{u}(p+k n)|^{2}|p+k n|^{2 t} \\
& \quad \leqq C n^{2 s-2 t} \sum_{k \in \mathbb{Z}^{*}}|\hat{u}(p+k n)|^{2}|p+k n|^{2 t},
\end{aligned}
$$

where we have used (2.12), the Schwarz inequality, (2.15a), (2.16) and (2.15d). From (2.20)-(2.22) we find that

$$
T_{4} \leqq C n^{2 s-2 t}\|u\|_{t}^{2}
$$

This completes the proof of (2.14) and so of Theorem 1.1 in the case $L=L_{0}$.

\section{Convergence of the Collocation Method for Operators with General Coefficients}

In this section we complete the proof of Theorem 1.1 by removing the restriction that the operators $L$ have constant coefficients, which was in force in the previous section (but still assuming a uniform mesh). The heart of the proof is a localization technique which enables us to deduce the convergence of the collocation method for the general operator $L$ from the convergence for certain constant coefficient operators derived from $L$ by freezing the coefficients $b_{+}$of its principal part. This technique, which hinges on a known commutation property of spline projections and multiplication by a smooth function and on well-known properties of pseudodifferential operators, is analogous to the familiar procedure in the theory of partial differential equation sometimes referred to as Korn's trick. Recently Prössdorf [33] has independently also applied this localization technique to the convergence theory for collocation methods.

For reference we recall the form (1.1) of the operator $L$ and its mapping properties:

$L$ is an isomorphism of $H^{r}$ onto $H^{r-\beta} \quad$ for all $r$

$Q_{+}^{\beta}$ and $Q_{-}^{\beta}$ map $H^{r}$ boundedly into $H^{r-\beta}$ for all $r$

$K$ maps $H^{r}$ boundedly into $H^{r-\beta+\delta} \quad$ for all $r$ and some $\delta>0$.

Also we state two lemmas, the first collecting known properties of finite elements applied to the periodic spline spaces, the second collecting known properties of pseudodifferential operators applied in our situation. We give proofs and references in the final section. 
Lemma 3.1. (a) (Approximation properties). Let $r<d+1 / 2$. Then there exists a family of approximation operators $P_{h}^{d}: H^{r} \rightarrow \mathscr{S}_{h}^{d}$ such that if $s \leqq r \leqq t \leqq d+1$ then

$$
\left\|u-P_{h}^{d} u\right\|_{s} \leqq C h^{t-s}\|u\|_{t}, \quad u \in H^{t},
$$

where $C$ does not depend on $h$ or $u$.

(b) The operators $P_{h}^{d}$ may be taken to have also the following additional property. If $\phi \in C^{\infty}$ then there exists a constant $C$ such that for all $v \in \mathscr{Y}_{h}^{d}$

$$
\left\|\phi v-P_{h}^{d}(\phi v)\right\|_{r} \leqq C h^{\delta}\|v\|_{r}, \quad \delta=\min (1, d+1-r) .
$$

(c) (Inverse properties). Let $s \leqq t<d+1 / 2$. Then there exists a constant $C$ such that

$$
\|v\|_{t} \leqq C h^{s-t}\|v\|_{s}, \quad v \in \mathscr{S}_{h}^{d} .
$$

(d) Let $s \leqq t<\alpha+1 / 2$. Then there exists a constant $C$ such that for all $u \in H^{t}$ and $v \in \mathscr{S}_{h}^{d}$

$$
\|u-v\|_{t} \leqq C\left(h^{s-t}\|u-v\|_{s}+\|u\|_{t}\right) .
$$

Lemma 3.2. (a) Let $\beta, t \in \mathbb{R}, \phi \in C^{\infty}, v \in H^{t}$. Then there exist constants $C$ and $q$ such that

$$
\left\|\phi\left(Q_{ \pm}^{\beta} v\right)-Q_{ \pm}^{\beta}(\phi v)\right\|_{t-\beta+1} \leqq C\|\phi\|_{q}\|v\|_{t}, \quad v \in H^{t} .
$$

Here $Q_{ \pm}^{\beta}$ may denote either of the operators $Q_{+}^{\beta}$ or $Q_{-}^{\beta}$.

(b) Let $t \in \mathbb{R}$ and $\phi, \psi \in C^{\infty}$, and suppose that $\phi \psi \equiv 0$. Then there exists a constant $C$ such that for all $v \in H^{t}$

$$
\left\|\phi Q_{ \pm}^{\beta}(\psi v)\right\|_{t-\beta} \leqq C\|v\|_{t-1} .
$$

(c) If $v \in H^{\beta}$ and $v \in H_{\mathrm{loc}}^{\beta+1}\left(I_{0}\right)$ for some closed interval $I_{0}$, then $Q_{ \pm}^{\beta} v \in H_{\mathrm{loc}}^{1}\left(I_{0}\right)$.

(d) Let $\beta, t \in \mathbb{R}, \varepsilon>0, b_{+}, b_{-} \in C^{\infty}$. Then there exists a $C^{\infty}$-partition of unity $\left\{\phi_{j}\right\}_{j=1}^{M}, C^{\infty}$ functions $\left\{\psi_{j}\right\}_{j=1}^{M}$ with $\left.\psi_{j}\right|_{\mathrm{supp} \phi_{j}} \equiv 1$, points $x_{j} \in \operatorname{supp} \phi_{j}$, and a constant $C$ such that for all $v \in H^{t}$

$$
\left\|\psi_{j}\left[b_{ \pm}-b_{ \pm}\left(x_{j}\right)\right] Q^{\beta} v\right\|_{t-\beta} \leqq \varepsilon\|v\|_{t}+C\|v\|_{t-1}, \quad j=1, \ldots, M .
$$

We now turn to the proof of Theorem 1.1. We separate out some important estimates in the following two propositions.

Proposition 3.3. Let $t, s \in \mathbb{R}$ satisfy $\beta \leqq s \leqq t<d+1 / 2$, and let $\phi \in C^{\infty}$. Then there exist positive constants $C_{1}, C$, and $\delta$ such that if $u \in H^{1}$ and $u_{h} \in \mathscr{S}_{h}^{d}$ then

$$
\begin{aligned}
\left\|\phi\left(u_{h}-u\right)\right\|_{t} \leqq & C_{1} h^{s-t}\left\|\phi L\left(u_{h}-u\right)\right\|_{s-\beta}+C\|u\|_{t}+C h^{s-t+\delta}\left\|u_{h}-u\right\|_{s} \\
& +\left\{\begin{array}{cc}
0 & s<t \\
C\left\|u_{h}-u\right\|_{s-\delta}, & s=t
\end{array}\right\} .
\end{aligned}
$$

Moreover the constant $C_{1}$ is independent of $\phi$.

Proof. Let $r=t$ and define projection operator $P_{h}^{d}$ as in Lemma 3.1. One easily verifies the decomposition 


$$
\begin{aligned}
\phi\left(u_{h}-u\right)= & \phi\left(P_{h}^{d} u-u\right)+P_{h}^{d} L^{-1} \phi L\left(u_{h}-P_{h}^{d} u\right) \\
& +\left(I-P_{h}^{d}\right) \phi\left(u_{h}-P_{h}^{d} u\right)+P_{h}^{d} L^{-1}(L \phi-\phi L)\left(u_{h}-P_{h}^{d} u\right) .
\end{aligned}
$$

We show that each of the four terms on the right hand side of (3.12) may be buunded in the $H^{t}$ norm by the right hand side of (3.11). Clearly

$$
\left\|\phi\left(P_{h}^{d} u-u\right)\right\|_{t} \leqq C\|u\|_{t} .
$$

For the second term we use the inverse property (3.6), the mapping properties of $L$ (3.1), the triangle inequality, and the approximation property (3.4) to get

$$
\begin{aligned}
\left\|P_{h}^{d} L^{-1} \phi L\left(u_{h}-P_{h}^{d} u\right)\right\|_{t} & \leqq C h^{s-t}\left\|L^{-1} \phi L\left(u_{h}-P_{h}^{d} u\right)\right\|_{s} \\
& \leqq C_{1} h^{s-t}\left\|\phi L\left(u_{h}-u\right)\right\|_{s-\beta}+C h^{s-t}\left\|u-P_{h}^{d} u\right\|_{s} \\
& \leqq C_{1} h^{s-t}\left\|\phi L\left(u_{h}-u\right)\right\|_{s-\beta}+C\|u\|_{t} .
\end{aligned}
$$

By (3.5), (3.7), and (3.4) the third term may be bounded in $H^{t}$ by

$$
C h^{\delta}\left\|u_{h}-P_{h}^{d} u\right\|_{t} \leqq C h^{s-t+\delta}\left\|u_{h}-u\right\|_{s}+C\|u\|_{t} .
$$

Finally we apply (3.4), (3.1), (3.8), (3.3), and (3.7) to get

$$
\begin{aligned}
& \left\|P_{h}^{d} L^{-1}(L \phi-\phi L)\left(u_{h}-P_{h}^{d} u\right)\right\|_{t} \\
& \quad \leqq C\left\|u_{h}-P_{h}^{d} u\right\|_{t-\delta_{1}} \leqq C\|u\|_{t}+ \begin{cases}C h^{s-t+\min \left(\delta_{1}, t-s\right)}\left\|u_{h}-u\right\|_{s}, & s<t, \\
C\left\|u_{h}-u\right\|_{s-\delta_{1}}, & s=t\end{cases}
\end{aligned}
$$

This completes the proof of the proposition.

Proposition 3.4. Let $t, s \in \mathbb{R}$ satisfy $t \neq \beta \leqq s \leqq t<d+1 / 2$. Then there exist positive constants $C$ and $\delta$ such that if $u \in H^{t}$ and $u_{h} \in \mathscr{Y}_{h}^{d}$ satisfy the collaction equations

then

$$
L u=L u_{h} \quad \text { on } \Delta,
$$

$$
\left\|u_{h}-u\right\|_{s} \leqq C h^{t-s}\|u\|_{t}+C h^{\delta}\left\|u_{h}-u\right\|_{s}+ \begin{cases}0, & s<t \\ C\left\|u_{h}-u\right\|_{s-\delta}, & s=t\end{cases}
$$

Proof. Again choose $P_{h}^{d}$ as in Lemma 3.1 with $r=t$ and choose $\left\{\phi_{j}, \psi_{j}, x_{j}\right\}_{j=1}^{M}$ as in Lemma $3.2 \mathrm{~d}$ with $\varepsilon$ to be specified below. Define

$$
L_{j}=b_{+}\left(x_{j}\right) Q_{+}^{\beta}+b_{-}\left(x_{j}\right) Q_{-}^{\beta}+\alpha J, \quad j=1, \ldots, M,
$$

with $\alpha=\gamma /(2 \pi)^{\beta / 2}$. From (2.2) we have

$$
C_{2}^{-1}\|v\|_{s+\beta} \leqq\left\|L_{j} v\right\|_{s} \leqq C_{2}\|v\|_{s+\beta}, \quad v \in H^{s}, s \in \mathbb{R},
$$

with $C_{2}$ depending only on $\beta, b_{+}$and $b_{-}$. Moreover if $w \in H^{t}$ and $w_{h} \in \mathscr{P}_{h}^{d}$ satisfy

$$
L_{j} w_{h}=L_{j} w \text { on } \Delta \text {, }
$$

then by the results of the previous section we have

$$
\left\|w-w_{h}\right\|_{s} \leqq C_{3} h^{t-s}\|w\|_{t}
$$


for $\beta \leqq s<d+1 / 2, s \leqq t \leqq d+1, \beta+1 / 2<t$, with $C_{3}$ depending only on $\beta, b_{+}, b_{-}$, $s$ and $t$ (but not on the choice of $\left\{\phi_{j}, \psi_{j}, x_{j}\right\}$ nor on $j$ ).

Now let $u$ and $u_{h}$ satisfy (3.13). Combining the identities

and

$$
L_{j} P_{h}^{d} \phi_{j} u_{h}=L_{j} \phi_{j} u+L_{j} \phi_{j}\left(u_{h}-u\right)+L_{j}\left(P_{h}^{d} \phi_{j} u_{h}-\phi_{j} u_{h}\right)
$$

$$
L_{j} \phi_{j}\left(u_{h}-u\right)=\left(L_{j}-L\right) \phi_{j}\left(u_{h}-u\right)+\left(L \phi_{j}-\phi_{j} L\right)\left(u_{h}-u\right)+\phi_{j} L\left(u_{h}-u\right)
$$

with (3.13), we get

where

$$
L_{j} P_{h}^{d} \phi_{j} u_{h}=L_{j} w_{j} \quad \text { on } \Delta
$$

$$
\begin{aligned}
w_{j}= & \phi_{j} u+L_{j}^{-1}\left(L_{j}-L\right) \phi_{j}\left(u_{h}-u\right) \\
& +L_{j}^{-1}\left(L \phi_{j}-\phi_{j} L\right)\left(u_{h}-u\right)+\left(P_{h}^{d}-I\right) \phi_{j} u_{h}
\end{aligned}
$$

or

$$
w_{j}=P_{h}^{d} \phi_{j} u_{h}-L_{j}^{-1} \phi_{j} L\left(u_{h}-u\right) \text {. }
$$

Applying (3.15) with $w=w_{j}$ and $w_{h}=P_{h}^{d} \phi_{j} u_{h}$ and using (3.18) we get

$$
\left\|L_{j}^{-1} \phi_{j} L\left(u_{h}-u\right)\right\|_{s} \leqq C_{3} h^{t-s}\left\|w_{j}\right\|_{t} .
$$

We now estimate $\left\|w_{j}\right\|_{t}$, bounding each of the four terms on the right hand side of (3.17) separately. Clearly

$$
\left\|\phi_{j} u\right\|_{t} \leqq C\|u\|_{t} .
$$

By (3.14) and the triangle inequality

$$
\begin{aligned}
T:= & \left\|L_{j}^{-1}\left(L_{j}-L\right) \phi_{j}\left(u_{h}-u\right)\right\|_{t} \\
\leqq & C_{2}\left[\left\|\psi_{j}\left(L_{j}-L\right) \phi_{j}\left(u_{h}-u\right)\right\|_{t-\beta}+\left\|\left(1-\psi_{j}\right)\left(L_{j}-L\right) \phi_{j}\left(u_{h}-u\right)\right\|_{t-\beta}\right] \\
\leqq & C_{2}\left[\left\|\psi_{j}\left(b_{+}\left(x_{j}\right)-b_{+}\right) Q_{+}^{\beta} \phi_{j}\left(u_{h}-u\right)\right\|_{t-\beta}\right. \\
& \left.+\left\|\psi_{j}\left(b_{-}\left(x_{j}\right)-b_{-}\right) Q_{-}^{\beta} \phi_{j}\left(u_{h}-u\right)\right\|_{t-\beta}\right] \\
& +C\left[\left\|u_{h}-u\right\|_{t-\delta}+\left\|\left(1-\psi_{j}\right)\left(L_{j}-L\right) \phi_{j}\left(u_{h}-u\right)\right\|_{t-\beta}\right] .
\end{aligned}
$$

Using (3.10) and (3.9) we deduce from (3.21) that

$$
T \leqq 2 C_{2} \varepsilon\left\|\phi_{j}\left(u_{h}-u\right)\right\|_{t}+C\left\|u_{h}-u\right\|_{t-\delta} .
$$

Using Proposition 3.3 and (3.14) to bound the first term we get finally

$$
T \leqq 2 C_{1} C_{2}^{2} \varepsilon h^{s-t}\left\|L_{j}^{-1} \phi_{j} L\left(u_{h}-u\right)\right\|_{s}+C\left[\|u\|_{t}+\left\|u_{h}-u\right\|_{t-\delta}+h^{s-t-\delta}\left\|u_{h}-u\right\|_{s}\right] .
$$

By (3.14), (3.8) and (3.3),

$$
\left\|L_{j}^{-1}\left(L \phi_{j}-\phi_{j} L\right)\left(u_{h}-u\right)\right\|_{\mathfrak{t}} \leqq C\left\|u_{h}-u\right\|_{t-\delta},
$$

and by (3.5), the triangle inequality, and (3.7), we find

$$
\left\|\left(P_{h}^{d}-I\right) \phi_{j} u_{h}\right\|_{t} \leqq C h^{\delta}\left\|u_{h}\right\|_{t} \leqq C\left(h^{s-t+\delta}\left\|u_{h}-u\right\|_{s}+h^{\delta}\|u\|_{t}\right) .
$$


Collecting these estimates together with (3.19) gives

$$
\begin{aligned}
\left\|L_{j}^{-1} \phi_{j} L\left(u_{h}-u\right)\right\|_{s} \leqq & 2 C_{1} C_{2}^{2} \varepsilon\left\|L_{j}^{-1} \phi_{j} L\left(u_{h}-u\right)\right\|_{s} \\
& +C\left[h^{t-s}\|u\|_{t}+h^{t-s}\left\|u_{h}-u\right\|_{t-\delta}+h^{s}\left\|u_{h}-u\right\|_{s}\right] .
\end{aligned}
$$

Selecting $\varepsilon=\left(4 C_{1} C_{2}^{2}\right)^{-1}$ we get

$$
\left\|L_{j}^{-1} \phi_{j} L\left(u_{h}-u\right)\right\|_{s} \leqq C\left[h^{t-s}\|u\|_{t}+h^{t-s}\left\|u_{h}-u\right\|_{t-\delta}+h^{\delta}\left\|u_{h}-u\right\|_{s}\right] .
$$

Since $\left\{\phi_{j}\right\}$ forms a partition of unity and $L$ is invertible,

$$
\begin{aligned}
\left\|u_{h}-u\right\|_{s} & \leqq C\left\|L\left(u_{h}-u\right)\right\|_{s-\beta} \leqq C \sum_{j=1}^{M}\left\|\phi_{j} L\left(u_{h}-u\right)\right\|_{s-\beta} \\
& \leqq C \sum_{j=1}^{M}\left\|L_{j}^{-1} \phi_{j} L\left(u_{h}-u\right)\right\|_{s} \\
& \leqq C\left[h^{t-s}\|u\|_{t}+h^{t-s}\left\|u_{h}-u\right\|_{t-\delta}+h^{\delta}\left\|u_{h}-u\right\|_{s}\right] .
\end{aligned}
$$

In case $s=t$, this completes the proof of the proposition. If $s<t$, we decrease $\delta$ in (3.22) so that $0<\delta \leqq s-t$ and use (3.7) (with $t$ replaced by $t-\delta$ ), to complete the proof.

We now complete the proof of Theorem 1.1. Assume momentarily that there exists $u_{h} \in \mathscr{S}_{d}^{h}$ satisfying (3.13) for some given $u \in H^{t}$. We shall prove that this implies the $(s, t)$-optimal convergence estimate

$$
\left\|u-u_{h}\right\|_{s} \leqq C h^{t-s}\|u\|_{t}
$$

at least for $h \leqq h_{0}$, where $h_{0}$ depends only on $L, s$, and $t$. Note that (3.23) implies that the homogeneous collocation equations admit only the trivial solution. Hence in general the solution $u_{h}$ to (3.13) exists and is uniquely determined. To prove (3.23) we distinguish three cases.

If $\beta \leqq s<t<d+1 / 2, t>\beta+1 / 2$, then (3.23) follows directly from Proposition 3.4 , for $h \leqq h_{0}$, with $h_{0}$ sufficiently small.

If $\beta+1 / 2<s=t<d+1 / 2$, we again use Proposition 3.4, replacing $\delta$ by $\min (\delta, s-\beta)$ so that we may assume from the outset that $\delta \leqq s-\beta$. Now applying the result of the previous paragraph with $s$ replaced by $s-\delta$, we get

$$
\left\|u-u_{h}\right\|_{s-\delta} \leqq C h^{\delta}\|u\|_{s} .
$$

Substituting (3.24) in Proposition 3.4 and choosing $h_{0}$ sufficiently small again gives (3.23) for $h \leqq h_{0}$.

Finally we consider the case $\beta \leqq s<d+1 / 2 \leqq t \leqq d+1$. Let us denote by $E_{h}$ the error operator $u \mapsto u_{h}-u$. Choose $t_{0} \in[s, d+1 / 2), t_{0}>\beta+1 / 2$. Then using the previous cases and Lemma 3.1 a (with $r=s$ ), we have

$$
\left\|E_{h}(u)\right\|_{s}=\inf _{\chi \in \mathscr{S}_{h}^{d}}\|E(u-\chi)\|_{s} \leqq C h^{t_{0}-s} \inf _{\chi \in \mathscr{S}_{h}^{d}}\|u-\chi\|_{t_{0}} \leqq C h^{t-s}\|u\|_{t},
$$

completing the proof. 


\section{Collocation on Smoothly Graded Meshes}

We now generalize Theorem 1.1 to allow a smoothly graded family of meshes in place of a family of uniform meshes. That is, we permit the family of meshes

$$
\phi^{-1}(\Xi)=\left\{\phi^{-1}(x) \mid x \in \Xi\right\} .
$$

Throughout this section $\phi$ denotes a strictly increasing function such that

$$
\phi(x+1)=\phi(x)+1
$$

(this condition insures that the mesh $\phi^{-1}(\Xi)$ repeats itself periodically). Moreover we assume that $\phi$ is a smooth diffeomorphism and the constants in the error bounds we prove here may depend on bounds for the derivatives of $\phi$ and $\phi^{-1}$. At present, however, we could not remove the theory of pseudodifferential operators from the proof of Theorem 4.2 and therefore require $C^{\infty}$.

Let $\mathscr{S}^{d}\left(\phi^{-1}(\Xi)\right)$ denote the space of smooth splines of degree $d$ on the mesh $\phi^{-1}(\Xi)$. The collocation problem is to find $u_{h} \in \mathscr{S}^{d}\left(\phi^{-1}(\Xi)\right)$ such that

$$
L u_{h}=F \quad \text { on } \phi^{-1}(\Delta) .
$$

The analogue of Theorem 1.1 regarding this method is

Theorem 4.1. Let $\beta, L, d$, and $F$ be as in Theorem 1.1. Then there exists $h_{0}>0$ such that (4.2) is uniquely solvable for $u_{h} \in \mathscr{S}^{\mathrm{d}}\left(\phi^{-1}(\Xi)\right)$ if $0<h \leqq h_{0}$. Moreover, if $s, t \in \mathbb{R}$ satisfy (1.5) and $u=L^{-1}(F) \in H^{t}$, then the error estimate (1.6) holds.

For the proof we require two lemmas. The first is essentially a case of the change of variable formula for pseudo-differential operators. The second states that the composition of a spline with a smooth function is itself almost a spline on the transformed mesh. It is analogous to Lemma $3.1 \mathrm{~b}$, which concerns the product of a spline with a smooth function. The proofs are postponed to the next section.

Lemma 4.2. The operator

$$
w \longmapsto\left[Q_{ \pm}^{\beta}(w \circ \phi)\right] \circ \phi^{-1}-\left(\phi^{\prime} \circ \phi^{-1}\right)^{\beta} Q_{ \pm}^{\beta} w
$$

maps $H^{t}$ boundedly into $H^{t-\beta+1}$ for all $t \in \mathbb{R}$.

Lemma 4.3. Let $r<d+1 / 2$. Then the operator $P_{h}^{d}$ of Lemma 3.1 may be taken so that for all $v \in \mathscr{S}^{d}\left(\phi^{-1}(\Xi)\right)$

$$
\left\|v \circ \phi^{-1}-P_{h}^{d}\left(v \circ \phi^{-1}\right)\right\|_{r} \leqq C h^{\delta}\|v\|_{r}, \quad \delta=\min (1, d+1-r) .
$$

Proof of Theorem 4.1. It suffices (as argued in the previous section), to prove that if $u_{h} \in \mathscr{S}^{d}\left(\phi^{-1}(\Xi)\right)$ satisfies

$$
L u_{h}=L u \quad \text { on } \phi^{-1}(\Delta),
$$

then (1.6) holds. Moreover if we prove (1.6) when $\beta \leqq s \leqq t<d+1 / 2$, the argument may be completed just as in the last paragraph of Sect. 3 . 
For $v$ in some $H^{r}$, set $\tilde{v}=v \circ \phi^{-1}$ and define $\tilde{L}: H^{r} \rightarrow H^{r-\beta}$ by

$$
\tilde{L} \tilde{v}=\tilde{L} v .
$$

By Lemma 4.2, $\tilde{L}$ is an operator of the form (1.1), and clearly (4.3) implies

Consequently,

$$
\tilde{L} \tilde{u}_{h}=\widetilde{L u} \text { on } \Delta \text {. }
$$

$$
\tilde{L}\left(P_{h}^{d} \tilde{u}_{h}\right)=\tilde{L}\left(P_{h}^{d} \tilde{u}_{h}-\tilde{u}_{h}+\tilde{u}\right) \quad \text { on } \Delta,
$$

where $P_{h}^{d}$ is given by Lemma 4.3.

Applying Theorem 1.1, we get

Now

$$
\left\|\tilde{u}-\tilde{u}_{h}\right\|_{s} \leqq C h^{t-s}\left\|P_{h}^{d} \tilde{u}_{h}-\tilde{u}_{h}+\tilde{u}\right\|_{t} .
$$

and

$$
\left\|u-u_{h}\right\|_{s} \leqq C\left\|\tilde{u}-\tilde{u}_{h}\right\|_{s}
$$

$$
\|\tilde{u}\|_{t} \leqq C\|u\|_{t} .
$$

Moreover, by Lemma 4.3, the triangle inequality, and Lemma 3.1d (which holds) for any quasiuniform mesh family),

$$
\left\|P_{h}^{d} \tilde{u}_{h}-\tilde{u}_{h}\right\|_{t} \leqq C h^{\delta}\left\|u_{h}\right\|_{t} \leqq C h^{\delta}\|u\|_{t}+C h^{s-t+\delta}\left\|u-u_{h}\right\|_{s} .
$$

Collecting these estimates and choosing $h_{0}$ sufficiently small proves (1.6) as desired.

\section{Proofs and References for Some Elementary Lemmas}

Proof of Lemma 2.3. First suppose that $d$ is odd. Then (2.9) may be written as

$$
\begin{aligned}
& f(y)=|y|^{d+1-\beta} \sum_{k=1}^{\infty}\left[(2 k-y)^{\beta-d-1}+(2 k+y)^{\beta-d-1}\right], \\
& g(y)=\operatorname{sign}(y)|y|^{d+1-\beta} \sum_{k=1}^{\infty}\left[(2 k-y)^{\beta-d-1}-(2 k+y)^{\beta-d-1}\right] .
\end{aligned}
$$

Since $\beta<d$ both series converge absolutely and uniformly for $y \in[-1,1]$. Moreover both functions are clearly even and bounded by a constant times $|y|^{d+1-\beta}$ and $f(0)=g(0)=0, g(1)=1$. Thus we need only show that both functions are strictly increasing on $[0,1]$. Since both series may be differentiated termwise we easily calculate for $y \in(0,1)$ that

$$
\begin{aligned}
& f^{\prime}(y)=2(d+1-\beta) y^{d-\beta} \sum_{k=1}^{\infty} k\left[(2 k-y)^{\beta-d-2}+(2 k+y)^{\beta-d-2}\right]>0, \\
& g^{\prime}(y)=2(d+1-\beta) y^{d-\beta} \sum_{k=1}^{\infty} k\left[(2 k-y)^{\beta-d-2}-(2 k+y)^{\beta-d-2}\right]>0,
\end{aligned}
$$

proving the lemma for $d$ odd. 
If $d$ is even (2.9) becomes

$$
\begin{aligned}
& f(y)=\operatorname{sign}(y)|y|^{d+1-\beta} \sum_{k=1}^{\infty}(-1)^{k+1}\left[(2 k-y)^{\beta-d-1}-(2 k+y)^{\beta-d-1}\right], \\
& g(y)=|y|^{d+1-\beta} \sum_{k=1}^{\infty}(-1)^{k+1}\left[(2 k-y)^{\beta-d-1}+(2 k+y)^{\beta-d-1}\right] .
\end{aligned}
$$

Now since $\beta<d+1$, the series $\sum_{k=1}^{\infty}(-1)^{k+1}(2 k \pm y)^{\beta-d-1}$ converges uniformly for $y \in[-1,1]$ and may be differentiated termwise. Therefore $f$ and $g$ define continuous even functions on $[-1,1]$ which are $O\left(|y|^{d+1-\beta}\right), f(0)=g(0)=0$, $g(1)=1$, and for $y \in(0,1)$,

$$
\begin{aligned}
f^{\prime}(y) & =2(d+1-\beta) y^{d-\beta} \sum_{k=1}^{\infty}(-1)^{k+1} k\left[(2 k-y)^{\beta-d-2}-(2 k+y)^{\beta-d-2}\right] \\
& \geqq 2(d+1-\beta) y^{d-\beta} \sum_{k=1}^{2}(-1)^{k+1} k\left[(2 k-y)^{\beta-d-2}-(2 \mathrm{k}-\mathrm{y})^{\beta-d-2}\right]>0, \\
g^{\prime}(y) & =2(d+1-\beta) y^{d-\beta} \sum_{k=1}^{\infty}(-1)^{k+1} k\left[(2 k-y)^{\beta-d-2}+(2 k+y)^{\beta-d-2}\right] \\
& \geqq 2(d+1-\beta) y^{d-\beta} \sum_{k=1}^{2}(-1)^{k+1} k\left[(2 k-y)^{\beta-d-2}+(2 k+y)^{\beta-d-2}\right]>0 .
\end{aligned}
$$

Thus $f$ and $g$ increase on $[0,1]$ and the lemma is proved.

Proof of Lemma 3.1. The inverse property stated in part (c) is well-known and requires only a quasiuniform mesh family. A reference which allows the full range of noninteger and negative indices is [18].

The approximation properties stated in part (a) are also familiar. In the uniform mesh case $P_{h}^{d}$ may be given constructively independent of $r$ (see [2]). Even on general meshes the result holds (see $[18,22])$. Approximation properties analogous to (3.5) have long been used in finite element analysis, cf., [29] Proposition 5, and [30] Hypothesis A.3. However to get this property in our periodic case involves a slight complication, so we outline the construction here.

For $a<b$ and $q$ real numbers, let $H^{q}(a, b)$ denote the nonperiodic Sobolev space, of index $q$ on the interval $(a, b)$. Also, for $q$ a positive integer we define the (periodic) piecewise Sobolev space $H^{q}(\Xi)$ consisting of those $u \in H^{q-1}$ such that $\left.u\right|_{\left(x_{j-1}, x_{j}\right)} \in H^{q}\left(x_{j-1}, x_{j}\right)$ where $\Xi=\left\{x_{j}\right\}$. For $u \in H^{q}(\Xi)$ we denote by $u(q) \in H^{0}$ the piecewise (not distributional) $q^{\text {th }}$ derivative. Finally let $x_{0} \in \Xi$ denote any nodal point. Since there is no distinction between periodic and nonperiodic splines of degree zero, we can find $P_{h}^{0}: H^{r-d}\left(x_{0}, x_{0}+1\right) \rightarrow \mathscr{S}_{h}^{0}$ such that if $s \leqq r-d \leqq t \leqq 1$

$$
\left\|u-P_{h}^{0} u\right\|_{s} \leqq C h^{t-s}\|u\|_{t}, \quad u \in H^{t}\left(x_{0}, x_{0}+1\right) .
$$

Since $P_{h}^{0}$ reproduces constants we have in particular that

$$
\left\|u-P_{h}^{0} u\right\|_{r-d} \leqq C h^{d+1-r}\left\|u^{\prime}\right\|_{L^{2}}, \quad u \in H^{1}\left(x_{0}, x_{0}+1\right) .
$$


Now if $u \in H^{1}(\Xi)$ we can choose $\psi \in \mathscr{S}_{h}^{0}$ such that $u+\psi \in H^{1}\left(x_{0}, x_{0}+1\right)$. Then by $(5.2)$

$$
\begin{aligned}
\left\|u-P_{h}^{0} u\right\|_{r-d} & =\left\|(u+\psi)-P_{h}^{0}(u+\psi)\right\|_{r-d} \leqq C h^{d+1-r}\left\|(u+\psi)^{\prime}\right\|_{L^{2}} \\
& \leqq C h^{d+1-r}\left\|u^{(1)}\right\|_{L^{2}},
\end{aligned}
$$

since $\psi^{(1)}=0$. (The trick of adding $\psi$ to cancel the discontinuities of $u$ was used in [17].)

Introduce now the map

$$
D g=g^{\prime}+\hat{g}(0)
$$

which defines an isometry of $H^{q}$ onto $H^{q-1}$ for all $q$. Set

$$
P_{h}^{d}=D^{-d} P_{h}^{0} D^{d}
$$

Then for $u \in H^{t}$ it follows immediately from (5.1) that for $s \leqq r \leqq t \leqq d+1$

$$
\begin{aligned}
\left\|u-P_{h}^{d} u\right\|_{s} & =\left\|D^{-d}\left(I-P_{h}^{0}\right) D^{d} u\right\|_{s}=\left\|\left(I-P_{h}^{0}\right) D^{d} u\right\|_{s-d} \\
& \leqq C h^{t-s}\left\|D^{d} u\right\|_{t-d} \leqq C h^{t-s}\|u\|_{t},
\end{aligned}
$$

verifying (3.4). Moreover if $u \in H^{(d+1)}(\Xi)$ then $D^{d} u \in H^{(1)}(\Xi)$ so (5.3) implies that

$$
\begin{aligned}
\left\|u-P_{h}^{d} u\right\|_{r} & =\left\|D^{-d}\left(I-P_{h}^{0}\right) D^{d} u\right\|_{r}=\left\|\left(I-P_{h}^{0}\right) D^{d} u\right\|_{r-d} \\
& \leqq C h^{d+1-r}\left\|\left(D^{d} u\right)^{(1)}\right\|_{L^{2}}=C h^{d+1-r}\left\|u^{(d+1)}\right\|_{L^{2}} .
\end{aligned}
$$

We apply this estimate to prove (3.5). For $v \in \mathscr{S}_{h}^{d}, \phi v \in H^{d+1}(\Xi)$, so

$$
\left\|\phi v-P_{h}^{d}(\phi v)\right\|_{r} \leqq C h^{d+1-r}\left\|(\phi v)^{(d+1)}\right\|_{L^{2}}
$$

Now since $v^{(d+1)}=0$, the Leibniz rule gives

$$
\left\|(\phi v)^{(d+1)}\right\| \leqq C\|v\|_{d}
$$

(where $C$ depends on $\phi$ ). Applying an inverse assumption,

$$
\|v\|_{d} \leqq C h^{\min (r-d, 0)}\|v\|_{r}
$$

and (3.5) follows.

It remains to prove part (d) of the lemma. This is a simple consequence of part (a) (with $r=t$ ) and part (c):

$$
\begin{aligned}
\|u-v\|_{t} & \leqq u-P_{h}^{d} u\left\|_{t}+\right\| v-P_{h}^{d} u\left\|_{t} \leqq C\right\| u\left\|_{t}+C h^{s-t}\right\| v-P_{h}^{d} u \|_{s} \\
& \leqq C\|u\|_{t}+C h^{s-t}\left[h^{t-s}\|u\|_{t}+\|v-u\|_{s}\right] .
\end{aligned}
$$

We now turn to the proof of Lemma 3.2. The results are all quite well-known. In particular part a) is a special case of the commutator property of pseudodifferential operator and parts b) and c) are weak forms of the pseudolocal property (see, e.g., $[44,47,48]$ ). Since we did not find statements in the literature easily applicable to our periodic case, we include brief elementary proofs. 
Proof of Lemma 3.2

a) Set $w=\phi\left(Q_{ \pm}^{\beta} v\right)-Q_{ \pm}^{\beta}(\phi v)$. Then

$$
\hat{w}(j)=\sum_{k \neq 0, j}\left[\sigma_{ \pm}(k)-\sigma_{ \pm}(j)\right] \hat{\phi}(j-k) \hat{v}(k)-\sigma_{ \pm}(j) \hat{\phi}(j) \hat{v}(0), \quad j \in \mathbb{Z}^{*},
$$

where $\sigma_{+}(k)=|k|^{\beta}, \sigma_{-}(k)=\operatorname{sign}(k)|k|^{\beta}$, so

$$
\begin{aligned}
\|w\|_{t-\beta+1}^{2}= & \sum_{j \neq 0}|2 \pi j|^{2(t-\beta+1)} \mid \sum_{k \neq 0, j}\left[\sigma_{ \pm}(k)-\sigma_{ \pm}(j)\right] \hat{\phi}(j-k) \hat{v}(k) \\
& -\left.\sigma_{ \pm}(j) \hat{\phi}(j) \hat{v}(0)\right|^{2}+|\hat{w}(0)|^{2} \\
\leqq & \left.\left.2 \sum_{j \neq 0}\left|\sum_{k \neq 0, j}\left\{\left|\sigma_{ \pm}(k)-\sigma_{ \pm}(j)\right||2 \pi j|^{t-\beta+1}|k|^{-t}|\hat{\phi}(j-k)|\right\}\right| k\right|^{t}|\hat{v}(k)|\right|^{2} \\
& +2\left[\sum_{j \neq 0}|2 \pi j|^{2(t-\beta+1)}|\hat{\phi}(j)|^{2}\right]|\hat{v}(0)|^{2}+|\hat{w}(0)|^{2} .
\end{aligned}
$$

We wish to bound the three terms on the right hand side on (5.6) by $C\|\phi\|_{q}^{2}\|v\|_{t}^{2}$. Now

$$
\left|\sigma_{ \pm}(k)-\sigma_{ \pm}(j)\right| \leqq \max (2,|\beta|)|k-j| \max \left(|k|^{\beta-1},|j|^{\beta-1}\right), \quad j, k \in \mathbb{Z}^{*},
$$

as follows from the mean value theorem, the triangle inequality, and - in the case of $\sigma_{-}-$a separate consideration of the case $k j<0$. Also

$$
|\hat{\phi}(m)| \leqq|2 \pi m|^{-\boldsymbol{r}}\|\phi\|_{r}, \quad m \in \mathbb{Z}^{*}, r \in \mathbb{R} .
$$

We now bound the bracketed term on the right hand side of (5.6). Employing (5.7) and (5.8) and the elementary inequality (a variant of Peetre's inequality)

$$
\frac{|j|^{r}|k|^{-r}}{|j-k|^{|r|}} \leqq 2^{|r|}, \quad r \in \mathbb{R}, j, k \in \mathbb{Z}^{*}, j \neq k,
$$

we get the bound $C\left\|_{i} \phi\right\|_{q} /|j-k|^{2}$ for appropriate constants $C$ and $q$ depending only on $\beta$ and $t$. Now

$$
\begin{aligned}
& \left.\sum_{j \neq 0}\left|\sum_{k \neq 0, j}\right| k\right|^{t}|\hat{v}(k)| /\left.|j-k|^{2}\right|^{2} \\
& \quad \leqq \sum_{j \neq 0}\left(\sum_{l \neq 0, j} \frac{1}{|j-l|^{2}}\right)\left(\sum_{k \neq 0, j} \frac{|k|^{2 t}|\hat{v}(k)|^{2}}{|j-k|^{2}}\right) \\
& \quad \leqq\left(\sum_{l \neq j} \frac{1}{|j-l|^{2}}\right)^{2} \sum_{k \neq 0}|k|^{2 t}|\hat{v}(k)|^{2} \leqq C\|v\|_{t}^{2} .
\end{aligned}
$$

Consequently the first term on the right hand side is bounded by $C\|\phi\|_{q}^{2}\|v\|_{t}^{2}$, as required. The second term may clearly be bounded similarly. Finally $|\hat{w}(0)|$ $=\left|\int \phi Q_{ \pm}^{\beta} v\right| \leqq\|\phi\|_{\beta-t}\|v\|_{t}$. Combining, (3.8) follows.

b) Since $\phi \psi=0$ we may apply (3.8) to get

$$
\left\|\phi Q_{ \pm}^{\beta}(\psi v)\right\|_{t-\beta}=\left\|\phi Q_{ \pm}^{\beta}(\psi v)-Q_{ \pm}^{\beta}[\phi(\psi v)]\right\|_{t-\beta} \leqq C\|\psi v\|_{t-1} \leqq C\|v\|_{t-1} .
$$


c) We must show that if $\phi \in C^{\infty}$ has support in $I_{0}$ (and its periodic translates) then $\phi Q^{\beta} v \in H^{1}$. Since $v \in H^{\beta+1}\left(I_{0}\right)$, then $\phi v \in H^{\beta+1}$, so $Q_{ \pm}^{\beta}(\phi v) \in H^{1}$. Also since $v \in H^{\beta}$, (3.8) implies that $\phi Q_{ \pm}^{\beta} v-Q_{ \pm}^{\beta}(\phi v) \in H^{1}$.

d) We require the following result:

For each $t \in \mathbb{R}$ there exist positive constants $C$ and $q$ such that

$$
\|\phi v\|_{t} \leqq\|\phi\|_{L^{\infty}}\|v\|_{t}+C\|\phi\|_{q}\|v\|_{t-1}
$$

for all $\phi \in C^{\infty}, v \in H^{t}$; cf. [45]. To prove (5.9) set $\Lambda^{t} v=(2 \pi)^{t} Q_{+}^{t} v+\hat{v}(0)$ so $\|v\|_{t}$ $=\left\|\Lambda^{t} v\right\|_{0}$. Hence $\|\phi v\|_{t} \leqq\left\|\phi \Lambda^{t} v\right\|_{0}+\left\|\Lambda^{t}(\phi v)-\phi \Lambda^{t} v\right\|_{0}$. Now

$$
\left\|\phi \Lambda^{t} v\right\|_{0} \leqq\|\phi\|_{L^{\infty}}\left\|\Lambda^{t} v\right\|_{0}=\|\phi\|_{L^{\infty}}\|v\|_{t},
$$

while (3.8) gives $\left\|\Lambda^{t}(\phi v)-\phi \Lambda^{t} v\right\|_{0} \leqq C\|\phi\|_{q}\|v\|_{t}$.

To complete the proof of d) we choose the partition of unity $\left\{\phi_{j}\right\}$ and functions $\psi_{j}$ so that $\left\|\psi_{j}\right\|_{L^{\infty}} \leqq 1$ and the support of each $\psi_{j}$ is small enough that $\left|b_{ \pm}(x)-b_{ \pm}(y)\right|<\varepsilon$ whenever $x, y \in \operatorname{supp} \psi_{j}$. Choosing any $x_{j} \in \operatorname{supp} \psi_{j}$ we have

$$
\left\|\psi_{j}\left[b_{ \pm}-b_{ \pm}\left(x_{j}\right)\right]\right\|_{L^{\infty}} \leqq \varepsilon,
$$

whence (3.10) follows from (5.9).

Proof of Lemma 4.2. The proposition of Lemma 4.2 is a special case of the symbol technique and asymptotic expansions in the theory of pseudo-differential operators since the operators

$$
\left[Q_{j}^{\beta}(w \circ \phi)\right] \circ \phi^{-1} \text { and }\left(\phi^{\prime} \circ \phi^{-1}\right)^{\beta} Q_{j}^{\beta}
$$

are pseudo-differential operators (see [40]) having the same principal symbols in each case $j= \pm$, respectively (see Theorem 5.1 in [47]). Then the mapping properties in Lemma 4.2 correspond to those of pseudo-differential operators of order $\beta-1$ in Sobolev spaces (Theorem 6.5 in [47]). The corresponding analysis rests on the fundamental and deep results on Fourier integral operators by Hörmander [23], and up to now we have not been able to find a simplified proof by using solely Fourier series and Fourier coefficients. Therefore we indicate here how for $j=+$ our operators can be identified with the corresponding Fourier integral operators and follow closely the presentation in [47, pp. 48-49] referring the reader for the detailed proofs there. (For $j=-$ one proceeds in the same manner.)

First note that for any 1-periodic function $u(x)$ the Fourier transform exists in the distributional sense taking the form

$$
F[u](s):=\int_{-\infty}^{+\infty} u(x) e^{-2 \pi i s x} d x=\frac{1}{2 \pi} \sum_{k \in \mathbf{Z}} \hat{u}(k) \delta(k-s)
$$

with $\delta$ the Dirac distribution and $s \in \mathbb{R}$. Thus, for periodic $u$,

$$
\begin{aligned}
Q_{+}^{\beta} u(y) & =\int_{\mathbb{R}} \sigma_{\beta+}(\xi) \sum_{k \in \mathbb{Z}} \hat{u}(k) \delta(k-\xi) e^{2 \pi i \xi y} d \xi \\
& =\int_{\mathbb{R}} \int_{\mathbb{R}}\left(2 \pi \sigma_{\beta+}(\xi)\right) u(y) e^{2 \pi i \xi(x-y)} d y d \xi
\end{aligned}
$$


where $\sigma_{\beta+}$ is a $C^{\infty}$-function with

This means

$$
\sigma_{\beta+}(\xi)= \begin{cases}|\xi|^{\beta} & \text { for }|\xi| \geqq 1 \text { and } \\ 0 & \text { for } \xi \text { near } 0\end{cases}
$$

$$
Q_{+}^{\beta} u=2 \pi \sigma_{\beta+}(D) u \quad \text { for every } 1 \text {-periodic distribution } u .
$$

By definition, $2 \pi \sigma_{\beta+}(D)$ is a classical pseudo-differential operator having the symbol $2 \pi \sigma_{\beta+}(\xi) \in S_{1.0}^{\beta}[47$, p. 37$]$. Now set

$$
t=\phi(x)=x+\phi^{(1)}(x)
$$

where $\phi^{(1)}(x)$ is 1 -periodic due to $(4.1)$, and

$$
x=\Psi(t):=\phi^{-1}(t), \quad \tau=\phi(y), y=\Psi(\tau) .
$$

Then

$$
\begin{aligned}
{\left[Q_{+}^{\beta}(w \circ \phi)\right] \circ \Psi(t) } & =\iint 2 \pi \sigma_{\beta+}(\xi) w(\phi(y)) e^{2 \pi i \xi(\Psi(t)-y)} d y d \xi \\
& =\iint 2 \pi \sigma_{\beta+}(\xi) w(\tau) e^{2 \pi i \xi(\Psi(\tau)-\Psi(\tau))} \Psi^{\prime}(\tau) d \xi d \tau
\end{aligned}
$$

Since the transformations defined by $\phi$ and $\Psi$, respectively, are supposed to be smooth and regular, we find that the functions

$$
\Phi(t, \tau):=\frac{\Psi(t)-\Psi(\tau)}{t-\tau} \text { and } 1 / \Phi
$$

are regular and positive for all $t$ and $\tau$. Hence, $\xi^{\prime}=\Phi \xi$ is for every $t, \tau$ a regular transformation and

with

$$
\left[Q_{+}^{\beta}(w \circ \phi)\right] \circ \Psi(t)=\int_{\mathbb{R}} \int_{\mathbb{R}} a\left(t, \tau, \xi^{\prime}\right) e^{2 \pi i \xi^{\prime}(t-t)} w(\tau) d \xi^{\prime} d \tau+K w
$$

$$
\begin{gathered}
a\left(t, \tau, \xi^{\prime}\right)=2 \pi \sigma_{\beta+}\left(\frac{\xi^{\prime}}{\Phi(t, \tau)}\right) \frac{\Psi^{\prime}(\tau)}{\Phi(t, \tau)} \Xi(|t-\tau|), \\
K w=\int_{\mathbb{R}} \int_{\mathbb{R}} 2 \pi \sigma_{\beta+}(\xi) w(\tau) e^{2 \pi i \xi\left(\Psi(t)-\Psi^{\prime}(\tau)\right)} \Psi^{\prime}(\tau) \cdot(1-\Xi(|t-\tau|)) d \xi d \tau,
\end{gathered}
$$

where $\Xi \in C_{0}^{\infty}(\mathbb{R})$ with $\Xi(\rho)=1$ for all $|\rho| \leqq 1$.

For the additional operator $K$ we find - by integration by parts -, that it can be written as the integral operator

$$
K w(t)=2 \pi \int_{y \in \mathbb{R}} w(\phi(y)) \frac{(1-\Xi(|t-\phi(y)|))}{(-2 \pi i(\Psi(t)-y))^{N}} \int_{\xi \in \mathbb{R}} \sigma_{\beta+}^{(N)}(\xi) e^{2 \pi i(\Psi(t)-y)} d \xi d y
$$

with $N \geqq \beta+2$ having a $C^{\infty}$-kernel. The first operator

$$
A w(t):=\int_{\mathbb{R}} \int_{\mathbb{R}} a\left(t, \tau, \xi^{\prime}\right) e^{2 \pi i \xi^{\prime}(t-\tau)} w(\tau) d \xi^{\prime} d \tau
$$

is a Fourier integral operator being "properly supported" whose amplitude function $a$ satisfies the estimates 


$$
\left|D_{\tau}^{\gamma} D_{t}^{\delta} D_{\xi^{\prime}}^{\alpha} a\left(t, \tau, \xi^{\prime}\right)\right| \leqq c_{\gamma \delta \alpha}\left(1+\left|\xi^{\prime}\right|\right)^{\beta-\alpha}
$$

for all $\alpha, \delta, \gamma \in \mathbb{N}_{0}$ and all $t, \tau, \xi^{\prime} \in \mathbb{R}$. Hence a belongs to Hörmander's class $S_{1,0,0}^{\beta}\left(\mathbb{R}^{3}\right),[47$, p. 43] and $A$ is a pseudo-differential operator which can be written as

$$
A w(t)=a(t, t, D) w+\iint b\left(t, \tau, \xi^{\prime}\right) e^{2 \pi i \xi^{\prime}(t-\tau)} w(\tau) d \xi^{\prime} d \tau
$$

where $b\left(t, \tau, \xi^{\prime}\right):=a\left(t, \tau, \xi^{\prime}\right)-a\left(t, t, \xi^{\prime}\right) \in S_{1,0,0}^{\beta-1}$. This is the decisive property which follows from Hörmander's results [23] (see Theorem 3.8, p. 44 in [47]).

$$
\begin{aligned}
a(t, t, D) w & =\iint 2 \pi \sigma_{\beta+}\left(\xi^{\prime} \phi^{\prime}(\Psi(t))\right) w(\tau) e^{2 \pi i \xi^{\prime}(t-\tau)} d \tau d \xi^{\prime} \\
& =\int_{\mathbb{R}} \sigma_{\beta+}\left(\xi^{\prime} \cdot \phi^{\prime}(\Psi(t)) \sum_{k \in \mathbf{Z}} \hat{w}(k) \delta\left(k-\xi^{\prime}\right) e^{2 \pi i \xi^{\prime} t} d \xi^{\prime}\right. \\
& =\left(\phi^{\prime} \circ \phi^{-1}\right)^{\beta} Q_{+}^{\beta} w
\end{aligned}
$$

for every 1-periodic function $w$. Since $K$ has $C^{\infty}$-kernel and $b \in S_{1,0,0}^{\beta-1}$, the proposition of Lemma 4.2 follows from the mapping properties of the Fourier integral operator with amplitude $b$, see Theorem 6.5 in [47].

Proof of Lemma 4.3. With the smoothness of $\phi^{-1}$, the mapping $D$ in (5.4), (5.5) and with the chain rule we find

$$
\begin{aligned}
\left\|v \circ \phi^{-1}-P_{h}^{d}\left(v \circ \phi^{-1}\right)\right\|_{r} & =\| D^{-d}\left(I-P_{h}^{0}\right) D^{d}\left(v \circ \phi^{-1} \|_{r}\right. \\
& =\left\|\left(I-P_{h}^{0}\right) D^{d}\left(v \circ \phi^{-1}\right)\right\|_{r-d} \\
& \leqq c h^{d+1-r}\left\|\left(v \circ \phi^{-1}\right)^{(d+1)}\right\|_{L^{2}(\Xi)} \\
& \leqq c h^{d+1-r} \sum_{j=0}^{d} c_{j}\left(\phi^{-1}\right)\left\|v^{(j)}\right\|_{L^{2}} \\
& \leqq c h^{d+1-r}\|v\|_{d},
\end{aligned}
$$

since $v^{(d+1)}=0$ between the break points. With an inverse assumptions as (3.6), Lemma 4.3 follows.

\section{References}

1. Agranovitch, M.S.: Spectral properties of elliptic pseudodifferential operators on a closed curve. Russ. Math. Surv. 13, 279-281 (1979)

2. Arnold, D.N.: A spline-trigonometric Galerkin method and an exponentially convergent boundary integral method. Math. Comput. 41, 383-397 (1983)

3. Arnold, D.N., Wendland, W.L.: On the asymptotic convergence of collocation methods. Math. Comput. 41, 349-381 (1983)

4. Atkinson, K.E.: A Survey of Numerical Methods for the Solution of Fredholm Integral Equations of the Second Kind. Philadelphia: Soc. Ind. Appl. Math. 1976

5. Atkinson, K.E., Graham, I., Sloan, I.: Piecewise continuous collocation for integral equations. SIAM J. Numer. Anal. 20, 172-186 (1983)

6. Banerjee, P.K., Butterfield, R.: Developments in Boundary Element Methods - 1. London: Appl. Sci. Publ. 1979

7. Banerjee, P.K., Shaw, R.P.: Developments in Boundary Element Methods - 2. London: Appl. Sci. Publ. 1982 
8. Bolteus, L., Tullberg, O.: BEMSTAT - A new type of boundary element program for twodimensional elasticity problems. In: Boundary Element Methods. C.A. Brebbia (ed.), pp. 518537. Berlin-Heidelberg-New York: Springer 1981

9. Brebbia, C.A.: Progress in Boundary Element Methods. London, Plymouth: Pentech Press 1981

10. Brebbia, C.A.: Boundary Element Methods. Berlin-Heidelberg-New York: Springer 1981

11. Brebbia, C.A.: Boundary Element Techniques, Methods in Engineering. Berlin-Heidelberg-New York: Springer 1983

12. Brebbia, C.A., Futagami, T., Tanaka, M.: Boundary Elements. Berlin-Heidelberg-New York: Springer 1983

13. Brebbia, C.A., Telles, J.C.F., Wrobel, L.C.: Boundary Element Techniques. Berlin-HeidelbergNew York: Springer 1984

14. Chatelin, F.: Spectral Approximation of Linear Operators. New York: Academic Press 1983

15. Crouch, S.L., Starfield, A.H.: Boundary Element Methods in Solid Mechanics. London: George Allen \& Unwin 1983

16. Cruse, T.A.: Application of the boundary-integral equation solution method in solid mechanics. In: Variational Methods in Engineering. Dept. Civil Eng. Southampton Univ., England, 9.19.29, 1972

17. Douglas, J., Jr., Dupont, T., Wahlbin, L.: Optimal $L_{\infty}$ error estimates for Galerkin approximations to solutions of two point boundary value problems. Math. Comput. 29, 475-583 (1975)

18. Elschner, J., Schmidt, G.: On spline interpolation in periodic Sobolev spaces, preprint P-Math01/83. Institut für Mathematik, Akademie der Wissenschaften der DDR, Berlin 1983

19. Filippi, P.: Theoretical Acoustics and Numerical Techniques. CISM Courses and Lectures 277. Wien-New York: Springer 1983

20. Hämmerlin, G., Schumaker, L.L.: Procedures for kernel approximation and solution of Fredholm integral equations of the second kind. Numer. Math. 34, 125-141 (1980)

21. Hayes, J.K., Kahaner, D.K., Kellner, R.G.: An improved method for numerical conformal mapping. Math. Comput. 26, 327-334 (1972)

22. Helfrich, H.-P.: Simultaneous approximation in negative norms of arbitrary order. R.A.I.R.O. Numer. Anal. 15, 231-235 (1981)

23. Hörmander, L.: Fourier integral operators I. Acta Math. 127, 79-183 (1971)

24. Hoidn, H.-P.: Die Kollokationsmethode angewandt auf die Symmsche Integralgleichung. Doctoral Thesis ETH Zürich, Switzerland, 1983

25. Hsiao, G.C., Kopp, P., Wendland, W.L.: Some applications of a Galerkin collocation method for integral equations of the first kind. Math. Meth. Appl. Sci. 6, 280-325 (1984)

26. Ivanov, V.V.: The Theory of Approximate Methods and their Application to the Numerical Solution of Singular Integral Equations. Leyden: Noordhoff Int. Publ. 1976

27. Mukherjee, S.: Boundary Element Methods in Creep and Fracture. London-New York: Applied Sci. Publ. 1982

28. Mustoe, G.G., Mathews, I.C.: Direct boundary integral methods, point collocation and variational procedures (To appear)

29. Nitsche, J., Schatz, A.: On local approximation properties of $L_{2}$-projections on spline subspaces. Appl. Anal. 2, 161-168 (1972)

30. Nitsche, J., Schatz, A.: Interior estimates for Ritz-Galerkin methods. Math. Comput. 28, 937958 (1974)

31. Noble, B.: Error analysis of collocation methods for solving Fredholm integral equations. In: Topics in Numerical Analysis. J.H. Miller (ed.), pp. 211-232. London: Academic Press 1972

32. Prenter, P.M.: A collocation method for the numerical solution of integral equations. SIAM J. Numer. Anal. 10, 570-581 (1973)

33. Prössdorf, S.: Ein Lokalisierungsprinzip in der Theorie der Spline-Approximationen und einige Anwendungen. Math. Nachr. 119, 239-255 (1984)

34. Prössdorf, S., Rathsfeld, A.: A spline collocation method for singular integral equations with piecewise continuous coefficients. Integral Equations Oper. Theory 7, 536-560 (1984)

35. Prössdorf, S., Rathsfeld, A.: On spline Galerkin methods for singular integral equations with piecewise continuous coefficients. (To appear in Numer. Math.)

36. Prössdorf, S., Schmidt, G.: A finite element collocation method for singular integral equations. Math. Nachr. 100, 33-60 (1981) 
37. Prössdorf, S., Schmidt, G.: A finite element collocation method for systems of singular integral equations. Preprint P-MATH-26/81, Institut für Mathematik, Akademie der Wissenschaften der DDR, Berlin 1981

38. Rizzo, F.J.: An integral equation approach to boundary value problems of classical elastostatics. Quart. Appl. Math. 25, 83-95 (1967)

39. Saranen, J., Wendland, W.L.: On the asymptotic convergence of collocation methods with spline functions of even degree. (Preprint 700, Math., Techn. Univ. Darmstadt 1982) (To appear in Math. Comput. 45 (1985))

40. Saranen, J., Wendland, W.L.: The Fourier series representation of pseudodifferential operators on closed curves. (In preparation)

41. Schmidt, G.: On spline collocation for singular integral equations. Math. Nachr. 111, 177-196 (1983)

42. Schmidt, G.: On spline collocation methods for boundary integral equations in the plane. (To appear in Math. Meth. Appl. Sci. 7 (1985))

43. Schmidt, G.: The convergence of Galerkin and collocation methods with splines for pseudodifferential equations on closed curves. Z. Anal. Anw. 3, 371-384 (1984)

44. Seeley, R.: Topics in pseudodifferential operators. In: Pseudo-Differential Operators. L. Nirenberg (ed.), pp. 169-305. Rome: Edizione Cremonese 1969

45. Strichartz, R.S.: Multipliers on fractional Sobolev spaces. J. Math. Mech. 16, 1031-1061 (1967)

46. Symm, G.T.: Integral equation methods in potential theory II. Proc. Royal Soc. London A 275 , 33-46 (1963)

47. Taylor, M.: Pseudodifferential Operators. Princeton: Princeton University Press 1981

48. Treves, F.: Introduction to Pseudodifferential and Fourier Integral Operators I. New YorkLondon: Plenum Press 1980

49. Watson, J.O.: Hermitian cubic boundary elements for plane problems of fracture mechanics. Res. Mechanica 4, 23-42 (1982)

50. Wendland, W.L.: Boundary element methods and their asymptotic convergence. In: Theoretical Acoustics and Numerical Techniques. P. Filippi (ed.), pp. 135-216. CISM courses and lectures 277. Wien-New York: Springer 1983

51. Quade, W., Collatz, L.: Zur Interpolationstheorie der reellen periodischen Funktionen. Sonderausgabe d. Sitzungsber. d. Preußischen Akad. d. Wiss., Phys.-math. K1., pp. 1-49. Berlin: Verlag d. Akad. d. Wiss. 1938

Received December 4, 1984/May 18, 1985 